\title{
An Ecological Approach to Software Supply Chain Risk Management
}

\author{
Sebastian Benthall ${ }^{\ddagger *}$, Travis Pinney ${ }^{\ddagger I I}, \mathrm{JC} \mathrm{Herz}^{* * \ddagger}$, Kit Plummer $\|^{\| \ddagger}$ \\ https: / / youtu.be/6UnuPhTPdnM
}

\begin{abstract}
We approach the problem of software assurance in a novel way inspired by an analytic framework used in natural hazard risk mitigation. Existing approaches to software assurance focus on evaluating individual software projects in isolation. We demonstrate a technique that evaluates an entire ecosystem of software projects, taking into account the dependencey structure between packages. Our model analytically separates vulnerability and exposure as elements of software risk, then makes minimal assumptions about the propagation of these values through a software supply chain. Combined with data collected from package management systems, our model indicates "hot spots" in the ecosystem of higher expected risk. We demonstrate this model using data collected from the Python Package Index (PyPI). Our results suggest that Zope and Plone related projects carry the highest risk of all PyPI packages because they are widely used and their core libraries are no longer maintained.
\end{abstract}

Index Terms-risk management, software dependencies, complex networks, software vulnerabilities, software security

\section{Introduction}

Systems that depend on complex software are open to many kinds of risk. One typical approach to software security that mitigates this risk is static analysis. We are developing novel methods to manage software risk through supply chain intelligence, with a focus on open source software ecosystems.

The Heartbleed bug in OpenSSL is an example of community failure and of how vulnerabilities in open source software can be a major security risk. [Wheeler2014] The recent failure of React, Babel, and many other npm packages due to the removal of one small dependency, left-pad, shows how dependencies can be a risk factor for production software. [Haney2016] These high profile examples, though quite different from each other, illustrate how software risk traverses the supply chain. As dependencies become more numerous and interlinked, the complexity of the system increases, as does the scope of risk management. Open source software projects make their source code and developer activity data openly available for analysis. This data can be used to mitigate software risk in ways that have not been explored.

\footnotetext{
* Corresponding author: sb@ischool.berkeley.edu

¥ Ion Channel, ionchannel.io

\& UC Berkeley School of Information

II travis.pinney@ionchannel.io

**jc.herz@ionchannel.io

II kit.plummer@ionchannel.io
}

Copyright $@ 2016$ Sebastian Benthall et al. This is an open-access article distributed under the terms of the Creative Commons Attribution License, which permits unrestricted use, distribution, and reproduction in any medium, provided the original author and source are credited.
With a small number of analytic assumptions about the propagation of vulnerability and exposure through the software dependency network, we have developed a model of ecosystem risk that predicts "hot spots" in need of more investment. In this paper, we demonstrate this model using real software dependency data extracted from PyPI using Ion Channel [IonChannel].

\section{Prior work}

[Verdon2004] outline the diversity of methods used for risk analysis in software design. Their emphasis is on architecturelevel analysis and its iterative role in software development. Security is achieved through managing information flows through architecturally distinct tiers of trust. They argue for a team-based approach with diverse knowledge and experience because "risk analysis is not a science". Contrary to this, our work develops a scientific theory of risk analysis, building on work from computer science and other fields.

There is a long history of security achieved through static analysis of source code. [Wagner2000] points out that the dependency of modern Internet systems on legacy code and the sheer complexity of source code involved makes manual source code level auditing very difficult. While some complex projects are audited by large and dedicated communities, not all software systems are so gifted in human resources. Therefore, static analysis tools based on firm mathematical foundations are significant for providing computer security at scale.

[Wheeler2015] develops a risk index for determining which open source software projects need security investments. This work is part of the Linux Foundation (LF) Core Infrastructure Initiative (CII) and published by the Institute for Defense Analysis. This metric is based on their expertise in software development analytics and an extensive literature review of scholarly and commercial work on the subject. They then apply this metric to Debian packages and have successfully identified projects needing investment. This work is available on-line as the CII Census project [CensusProject].

While software security studies general focus on the possibility of technical failure of software systems, open source software exposes an additional risk of community failure. Development of a software project may cease before it reaches a state of usability and maturity. [Schweik2012] is a comprehensive study of the success and failure of open source projects based on large-scale analysis of SourceForge data, as well as survey and interview data. They define a successful project as one that performs a 
useful function and has had at least three releases. They identify several key predictive factors to project success, including data that indicates usefulness (such as number of downloads), number of hours contributed to the project, and communicativeness of the project leader.

These precedents focus on individual software projects and their susceptiblity to technical and community failure. [Nagappan2005] and [Nagappan2007] look at dependency relationships between packages and specifically relative code churn (changes in lines of code) between dependent packages as a cause of system defects in Windows Server 2003.

We build on these approaches by considering security as a function of the entire software supply chain. This supply chain resembles a complex ecosystem more than a simple 'chain' or stack. We draw inspiration from a risk management strategy approaches used in another kind of complex system, namely disaster risk reduction and climate change adaptation research developed by Cardona [Cardona2012] and widely used by the World Bank's Global Facility for Disaster Risk Reduction among others [Yamin2013].

This framework evaluates the expected cost of low-probability events by distinguishing three factors of risk: hazards, exposure, and vulnerabilities. Hazards are potentially damaging factors from the environment. Exposure refers to the inventory of elements in places where hazards occur. Vulnerabilities are defined as the propensity of exposed elements to suffer adverse effects when impacted by a hazard. Expected risk is then straightforwardly calculated using the formula:

$$
\text { risk }=\text { hazard } * \text { vulnerability } * \text { exposure }
$$

We adapting this framework to cybersecurity in the software ecosystem. There are significant differences between modeling risk from natural hazards and modeling cybersecurity risk. Most notably, cybersecurity threats can be deliberately adversarial, detecting and exploiting specific weaknesses rather than presenting a general hazard. In this work we focus on the interplay between exposure and vulnerability in the software ecosystem and abstract away the specificity of a threat model. We see an analytic treatment of that interplay as a valuable step in tractable security analysis of the software supply chain.

\section{Modeling Ecological Risk in Software}

\section{Software dependency and project risk}

Some previous studies of software risk [Wheeler2015] have suffered from the ambiguity of how 'risk' is used in a software development context. Security research often contextualizes problems within a specific threat model. But for some applications, such as identifying software projects in need of additional investment in order to mitigate risk from generalized and potentially unknown threats, this kind of threat modeling is inappropriate. A general concern with supply chain security motivates a different approach.

If we break down the sources of risk and how these affect the need for security investments analytically, we can distinguish between several different factors:

- Vulnerability. A software project's vulnerability is its intrinsic susceptibility to attack. Common Vulnerability and Exposure (CVE) records are good examples of specific software vulnerabilities. But software's vulnerability can also be predicted from a general property, such as the language it's written in. (Some languages, such as $\mathrm{C}++$, are harder to write in securely and therefore generally more vulnerable [Wheeler2015])

- Exposure. A software project's exposure is its extrinsic availability to attack. A direct network connection is a source of exposure.

Vulnerability and exposure are distinct elements of a software project's risk. Analyzing them separately and then combining them in a principled way gives us a better understanding of a project's risk.

Dependencies complicate the way we think about vulnerability and exposure. A software project doesn't just include the code in its own repository; it also includes the code of all of its dependencies, often tied to a specific version. Furthermore, a package does not need to be installed directly to be exposed--it can be installed as a dependency of another project, or as a transitive dependency. Based on these observations, we can articulate two heuristics for use of dependency topology in assessing project risk:

- If $\mathrm{A}$ depends on $\mathrm{B}$, then a vulnerability in $\mathrm{B}$ implies a corresponding vulnerability in A.

- If $\mathrm{A}$ depends on $\mathrm{B}$, then an exposure to $\mathrm{A}$ implies an exposure to $\mathrm{B}$.

For example, if a web application (A) uses a generic web application framework (B), and that web application is installed and recieving web traffic, then there is an instance of the web framework installed and recieving web traffic. The framework is exposed through the web application. If there is a vulnerability in the web application framework (such as a susceptibility to SQL injection attacks), then the web application will inherit that vulnerability. There are exceptions to these rules. Developers of the web application (A) might recognize the vulnerability to SQL injection and fix the problem without pushing the change upstream (to B). Nevertheless, this is a principled analytic way of relating vulnerability, exposure, and software dependency that can be implemented as a heuristic and tested as a hypothesis.

The risk analysis framework described above is very general. Due to this generality, the framework suffers from the ambiguity of its terms. Depending on the application of this framework, "vulnerability" refers to literal software vulnerabilities such as would be reported in a CVE. When we analyze the software ecosystem as a supply chain, we are often concerned about higher level properties that serve as general proxies for whole classes of error or failure.

\section{Robustness and resilience}

We find the distinction between system robustness and system resilience helpful. We define the robustness of a system as its invulnerability to threats and hazards, as a function of its current state. We define the resilience of a system as its capacity to recover quickly from injury or failure. A mature, well-tested system will be robust. A system with an active community ready to respond to the discovered of a new exploit will be resilient.

A system can be robust, or resilient, both, or neither. Robustness and resilience can be in tension with each other. For example, the more churn a software project is, measured as a function of the activity of the community and frequency of new commits, the more likely that it will be resilient, responding to new threat information. But it is also likely to be less robust, as new code might introduce new software flaws. [Nagappan2005] and 
[Nagappan2007] find that relative code churn between dependent packages is a significant predictor of system defects.

We refer to a system that is not robust as fragile, and a system that is not resilient as brittle. Fragility and brittleness are two distinct and general ways in which a component of a software ecosystem might be vulnerable.

\section{Computing fragility and exposure}

Our risk analysis framework defines exposure and vulnerability as abstract components of risk that can be defined depending on the hazards and threats under consideration. In the example of this study, we will define these variables with an interest in the general prediction of robustness in widely used software. This sort of analysis would be useful in determining which software packages are in need of further investment in order to reduce risk globally.

In the following analysis, we will define exposure to be the number of times a package has been downloaded. We assume for the sake of this analysis that more widely downloaded software is more widely used and exposed to threats. This metadata is provided by PyPI for each package directly.

We will define vulnerability specifically in terms of software fragility, and make the assumption that frequently released software is less fragile. While it is true that sometimes a new software release can introduce new flaws into software, we assume that, on average, more releases mean a more active community, more robust development processes, and greater maturity in the project lifecycle. Specifically for the purpose of this study we will define

$$
\text { fragility }(p)=\frac{1}{\text { number_of_releases }(p)}
$$

In future work, we will revise and validate these metrics.

\section{Implementation of risk computation}

The risk analysis framework presented in the above section Software dependency and project risk is designed to be widely applicable, factoring risk into abstract exposure and vulnerability factors and then making minimal assumptions about how these factors propagate through the dependency graph.

In practice, the application of this framework will depend on the selection of package metadata used to measure exposure and vulnerability. Below is a Python implementation of efficient risk computation using a directed graph representation of package dependencies and NetworkX. [Hagberg2008] It imports data as a graph, where packages are nodes, directed edges indicate package dependencies, and relevant metadata are precomputed properties of the nodes. In this code, we use a precomputed 'fragility' metric as the vulnerability variable, and the number of unique downloads of each package as the exposure variable. Running this code imports the data from a Graph Exchange XML Format (GEXF) file, computes the ecosystem risk of each package, and exports the data to a different file.

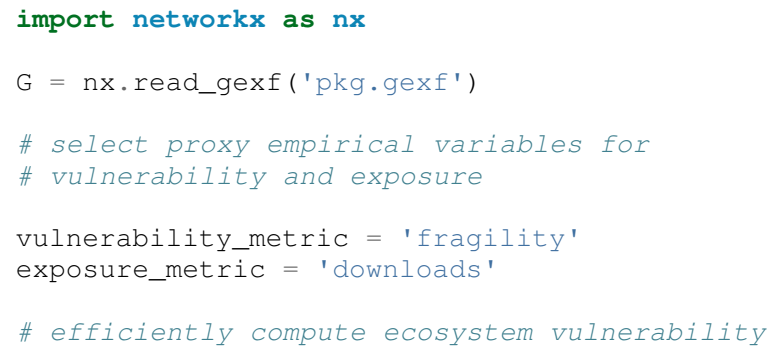

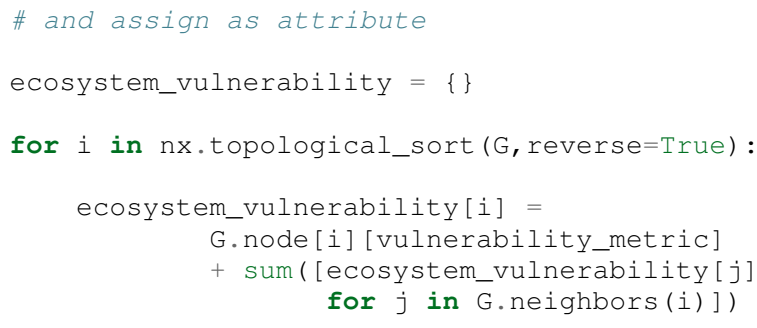

A significant problem with this implementation of risk calculation is that if node $\mathrm{A}$ is accessible to node $\mathrm{B}$ through multiple distinct paths, then the vulnerability (or exposure) of $\mathrm{B}$ will be counted towards A's ecosystem vulnerability (or exposure) once for each path. A superior version of this algorithm would ensure that each node was only counted once in ecosystem measurements. The version of the algorithm presented above uses a heuristic measure for performance reasons.

\section{Removing cycles}

The above algorithm has one very important limitation: it assumes that there are no cycles in the dependency graph. This property is necessary for the nodes to have a well-defined topological order. However, Python package dependencies do indeed include many cycles. An amusing example are the packages chicken and egg. We can adapt any directed cyclic graph into a directed acyclic graph simply by removing one edge from every cycle.

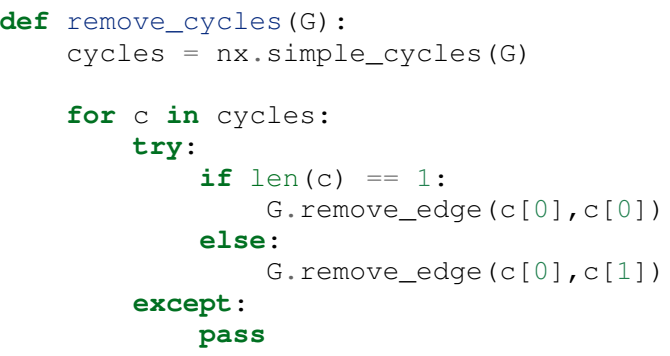


One way to improve this algorithm would be to remove as few edges as possible in order to eliminate all cycles. Another way to improve this algorithm would be to adapt the heuristic assumptions that motivate this framework to make reasonable allowances for cycle dependencies. It is unknown how these changes will effect the results. We leave the elaboration of this algorithm for future work.

\section{Data collection and publication}

Data for this analysis comes from two sources. For package and release metadata, we used data requested from PyPI, the Python Package Index. This includes the publication date and number of unique downloads for each software release. We also downloaded each Python release and inspected it for the presence of a setup.py file. We then extracted package dependency information from setup.py through its install_requires field. This data is available in . gexf format [Benthall2016].

Python dependencies are determined through execution of Python install scripts. Therefore, our method of discovering package dependencies via static analysis of the source code does not capture all cases.

For each package, we consider dependencies to be the recursive union of all requirements for all releases. Specifically we collapse all releases of a package into a single node in the dependency graph. While this loses some of the available information, it is sufficient for this prelimenary analysis of the PyPI ecosystem.

\section{Empirical and Modeling Results}

Our data collection process created a network with 66,536 nodes and 72,939 edges. Over half of the nodes, 33,573, have no edge. This isolates them from the dependency network. Of the remaining $32,963,31,473$ belong to a single giant connected component. Complex networks often exhibit the preponderance of a single connected component like this.

\section{Statistical properties of the software dependency network}

The PyPI package dependency network resembles classical complex networks, with some notable departures.

A early claim in complex network theory by [Newman2002], [Newman2003] is that random complex networks will exhibit negative degree assortativity, and that social networks will exhibit positive degree assortativity due to homophily or other effects of group membership on network growth. [Noldus2015] notes that in directed graphs, there are four variations on the degree assortativity metric as for each pair of adjacent nodes one can consider each node's in-degree and out-degree. The degree assortativity metrics for the PyPI dependency graph are given in Table 1.

The PyPI package dependency network notably has in-in degree assortativity of 0.19 , and out-in degree assortativity of -0.16 . The in-out and out-out degree assortativities are both close to zero. We have constructed the graph with the semantics that an edge from $\mathrm{A}$ to $\mathrm{B}$ implies that $\mathrm{A}$ depends on $\mathrm{B}$.

This is a strange structure because its assortativity measures defy the assortativity patterns seen in other complex networks. One reason is that there is much greater variation in out-degree than in in-degree. Table 2 shows the top ten most depended on packages. Table 3 shows the top ten packages with the most dependencies. Three packages, requests, six, and django have out-degree over 1000.

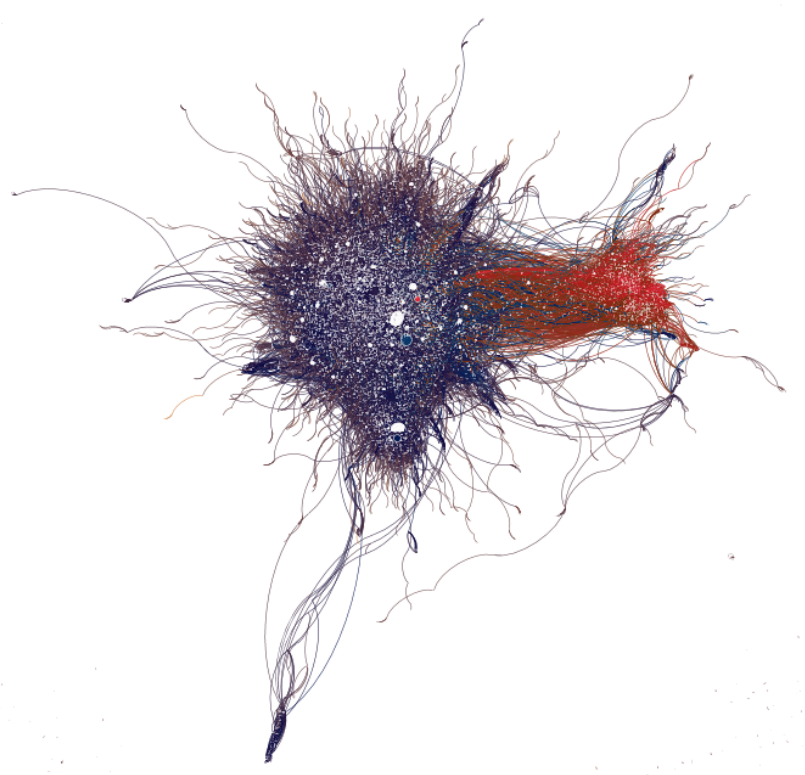

Fig. 1: Visualization of PyPI dependency network. Annotated dependency graph (see Implementation of risk computation) was exported as .gexf, loaded into Gephi [Bastian2009], styled using Force Atlas layout, and colored by ecosystem risk property. This visualization does not include singleton nodes with zero degree, which are the vast majority of nodes. Node size is proportional to out degree. Nodes are colored by the log (base 10) of package ecosystem risk. Red nodes are higher risk. The large red cluster consists of projects related to the Zope web application server, including the Plone content management system.

\begin{tabular}{ll}
\hline Metric & Value \\
\hline in-in & 0.19 \\
in-out & 0.05 \\
out-in & -0.16 \\
out-out & -0.04 \\
\hline
\end{tabular}

TABLE 1: Degree assortativity metrics for the PyPI dependency graph.

\section{Hot spot analysis}

Our analysis suggests that the riskiest packages in the Python ecosystem are those that are part of the Zope web application server and the Plone content management system (CMS) built on it. The Zope community has declared that Zope is now a legacy system and does not recommend that developers use these projects. Therefore, our analytic findings are consistent with community and domain knowledge regarding the resilience of these communities. Despite these warnings, the Plone community is still active and many web sites may still depend on this legacy technology. This study motivates further work on the resilience of Zope to new security threats.

The security properties of Plone have been the subject of considerable informal debate. [Walsh2011] noted that Plone has an order of magnitude lower number of vulnerabilites reported in Mitre's Common Vulnerabilities and Exposures database compared to other popular CMSes like Joomla, Drupal, and Word- 


\begin{tabular}{ll}
\hline Package & Out-Degree \\
\hline requests & 2125 \\
six & 1381 \\
django & 1174 \\
pyyaml & 775 \\
zope.interface & 663 \\
lxml & 619 \\
flask & 607 \\
python-dateutil & 599 \\
zope.component & 550 \\
jinja2 & 507 \\
\hline
\end{tabular}

TABLE 2: Top ten most dependencies.

\begin{tabular}{ll}
\hline Package & Out-Degree \\
\hline plone & 92 \\
mypypi & 53 \\
invenio & 52 \\
ztfy.sendit & 48 \\
ztfy.blog & 47 \\
smartybot & 47 \\
icemac.addressbook 41 \\
sentry & 40 \\
products.silva & 38 \\
ztfy.scheduler & 37 \\
\hline
\end{tabular}

TABLE 3: Top ten packages by number of dependencies.

press. This has lead Wikipedia [Wiki2016] to assert that Plone's security record is cause of its widespread adoption by government and non-government organizations. [Byrne2013] has challenged this conventional wisdom, noting that the high number of recorded vulnerabilites may just as likely be due to the much greater popularity of the other CMS's. That Drupal, Wordpress, and Joomla are all written in PHP is another confounding factor, as PHP may

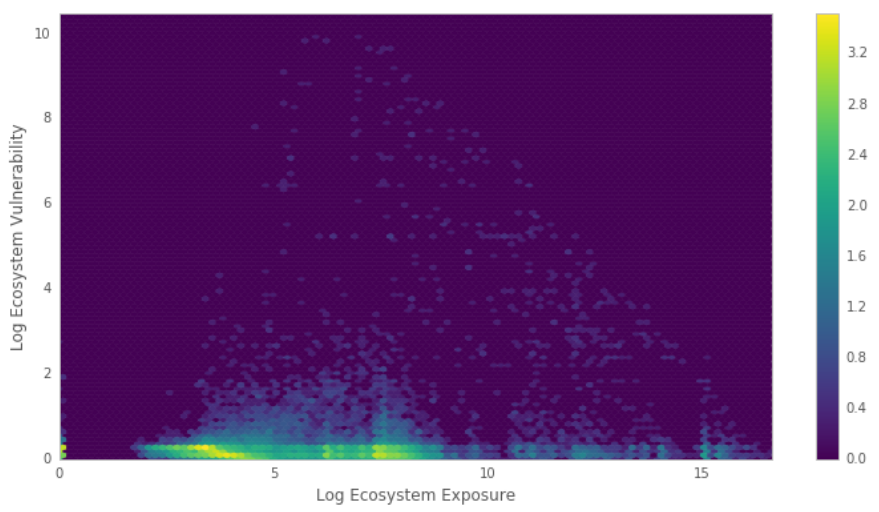

Fig. 2: Hex plot of log vulnerability and log exposure of each package, with bin density scored on log scale. All logs are base 10. Exposure is more widely distributed than vulnerability. Vulnerability scores for the vast majority of packages are low. There is a fringe of packages that are either highly vulnerable, highly exposed, or both. There is a loglinear tradeoff between high vulnerability and high exposure. This is most likely due to the fact that ecosystem vulnerability and ecosystem exposure both depend on an package's position in the dependency network. Rendered with Matplotlib [Hunter2007]. be a language prone to security problems. Drupal, Joomla, and Wordpress are beyond the scope of our study, which is concerned only with the PyPI ecosystem. In our risk analysis, Plone scores poorly compared to other Python web frameworks such as Django and Flask. We take this as an indication that beyond its scientific merits, our risk analysis method can provide actionable insights into security that are relevant to practicing software engineers.

We have also identified six, a Python 2 and Python 3 compatibility library, as an ecosystem risk hot spot. The second most depended on project in PyPI, six inherits its exposure from all of its downstream descendants. For this reason, it is important to ensure that six does not have any security-related flaws.

We must admit that there is another reason why the Plone ecosystem has score highly in software risk. The Zope and Plone packages are notably dense in their dependency connectivity. In the original dependency network, before cycles were excised from the graph by removing edges, many Zope and Plone packages were implicated in large cycles of mutual dependency. Even with many of these edges removed, it is possible that packages in this subsystem are more likely to be linked by multiple disitinct paths. With our present algorithm, this would result in some packages being double counted. Due to this technical complication, we must conclude that our results, though suggestive, are only tentative pending future work.

\section{Discussion and future work}

We have synthesized techniques from computer security and disaster risk reduction to develop a novel method of predicting risk in the software ecosystem. This fits within the broad scope of supply chain analysis, though we recognize that the software ecosystem as a whole is not merely a chain, but a complex network with a distinctive topology. We approach risk analysis as a science that employs static analysis techniques but also looks more broadly at developer communities and the rate and flow of their activities and communications. This paper proposes a framework of predicting risk in software infrastructure based on static analysis of package dependencies, metadata about downloads and release schedules, and minimal assumptions about the distribution of exposure and vulnerability in software. We have demonstrated the implications of this framework using the PyPI package ecosystem.

A major shortcoming of our analysis is the lack of validation against a gold standard data of ground truth regarding software risk. In future work, we will test this framework using other data sets, including data from project issue trackers (such as GitHub) and Common Vulnerabilities and Exposure (CVE) data. We anticipate that linking this data with package dependencies will require a non-trivial amount of work on entity resolution. It is an open question to what extent this framework is useful for assessing software robustness (absence of software errors that can be exploited, for example) and software resilience (capacity of software development communities to respond to known exploits).

There is also room to improve our data preprocessing in future work. For the work in this paper, Python dependencies were discovered using crude static analysis. We used a regular expression to parse each package's setup. py file. Python requirements are in fact determined upon package installation by executing Python code. We can get more accurate data by running the setup scripts and extracting requirements from the resulting Python objects.

We simplified the dependency graph by considering any requirement relation between any versions of two packages to be 


\begin{tabular}{|c|c|c|c|c|c|c|c|c|}
\hline & Log Eco. Risk & Log Eco. Vulnerability & Log Eco. Exposure & Fragility & Num. Releases & Downloads & In Degree & Out Degree \\
\hline zope.app.publisher & 17.54 & 6.95 & 10.59 & 0.04 & 26 & 232460 & 24 & 54 \\
\hline zope.app.form & 17.54 & 6.89 & 10.64 & 0.04 & 26 & 265370 & 19 & 45 \\
\hline five.formlib & 17.44 & 6.47 & 10.97 & 0.20 & 5 & 127280 & 13 & 10 \\
\hline plone & 17.44 & 2.37 & 15.07 & 0.01 & 79 & 387614 & 96 & 152 \\
\hline zope.interface & 17.42 & 10.48 & 6.94 & 0.03 & 31 & 8685819 & 0 & 841 \\
\hline zope2 & 17.41 & 6.40 & 11.01 & 0.03 & 32 & 241354 & 28 & 163 \\
\hline zope.traversing & 17.32 & 8.40 & 8.92 & 0.04 & 28 & 367494 & 9 & 181 \\
\hline zope.schema & 17.29 & 9.61 & 7.68 & 0.03 & 31 & 624429 & 4 & 399 \\
\hline zope.site & 17.28 & 7.60 & 9.68 & 0.07 & 14 & 255063 & 9 & 72 \\
\hline zope.container & 17.27 & 7.73 & 9.54 & 0.05 & 20 & 294873 & 20 & 119 \\
\hline
\end{tabular}

TABLE 4: Highest risk Python packages. All logs base 10.

sufficient for an edge in the final graph. In reality, package requirements configurations often refer to specific versions or version ranges in their dependencies. In order to take this into account, we will need to reexamine our risk model and its assumptions about vulnerability and exposure propagation. A fully dynamic version of our risk model would also take into account how proxy variables such as number of unique downloads change between versions.

The research presented here deals exclusively with data about technical organization. However, as we expand into research into how software communities and their interactions are predictive of software risk, we must be mindful of ethical considerations. Though all the data we intend to use is public and more importantly known to be public in the context of software development, study of human subjects is nevertheless sensitive. Our research agenda depends critically on maintaining the trust of the developer communities we study. For this reason we are dedicated to ecosystems and software projects, which aggregate individual efforts, as the fundamental unit of analysis.

\section{Acknowledgements}

We gratefully acknowledge David Lippa, Kyle Niemeyer, and J. Edward Pickle for their helpful comments.

\section{References}

[Bastian2009] Bastian, Mathieu, Sebastien Heymann, and Mathieu Jacomy. "Gephi: an open source software for exploring and manipulating networks." ICWSM 8 (2009): 361362.

[Benthall2016] Sebastian Benthall. (2016). PyPI Packages Annotated. Zenodo. 10.5281/zenodo.57563

[Byrne2013] Byrne, Tony. "Is Plone Really More Secure Than Drupal and Joomla?" Web log post. Real Story Group. N.p., 11 Feb. 2013. Web. 23 June 2016.

[Clauset2007] A. Clauset, C.R. Shalizi, and M.E.J. Newman. Powerlaw distributions in empirical data. arXiv:0706.1062, June 2007.

[Mitzenmacher2003] Mitzenmacher, M. 2003. "A Brief History of Generative Models for Power Law and Lognormal Distributions." Internet Mathematics Vol. 1, No. 2: 226-251

[CensusProject] Census Project. (n.d.). Retrieved July 12, 2016, from https://www.coreinfrastructure.org/programs/censusproject

[Cardona2012] Cardona, Omar-Daria, et al. "Determinants of risk: exposure and vulnerability." (2012).

[Girardot2013]
[Hagberg2008]

[Hunter2007]

[IonChannel]

[LaBelle2004]

[Nagappan2005]

[Nagappan2007]

[Newman2002]

[Newman2003]

[Noldus2015]

[Schweik2012]

[Verdon2004]

[Walsh2011]

[Wagner2000]

[Wiki2016]

[Wheeler2015]

[Yamin2013]
Aric A. Hagberg, Daniel A. Schult and Pieter J. Swart, "Exploring network structure, dynamics, and function using NetworkX", in Proceedings of the 7th Python in Science Conference (SciPy2008), Gäel Varoquaux, Travis Vaught, and Jarrod Millman (Eds), (Pasadena, CA USA), pp. 11-15, Aug 2008

David Haney. 2016. "NPM \& left-pad: Have We Forgotten How To Program?" http://www.haneycodes.net/ npm-left-pad-have-we-forgotten-how-to-program/

Hunter, J. D. (2007). Matplotlib: A 2D graphics environment. Computing in science and engineering, 9(3), 90-95. http://dx.doi.org/10.5281/zenodo.44579 io/

N. LaBelle, E. Wallingford. 2004. Inter-package dependency networks in open-source software. Nagappan, N., \& Ball, T. (2005, May). Use of relative code churn measures to predict system defect density. In Proceedings. 27th International Conference on Software Engineering, 2005. ICSE 2005. (pp. 284-292). IEEE.

Nagappan, N., \& Ball, T. (2007). Explaining failures using software dependences and churn metrics. In Proceedings of the 1st International Symposium on Empirical Software Engineering and Measurement.

Newman, M. E. J. 2002. "Assortative mixing in networks."

Newman, M. E. J. 2003. "Mixing patterns in networks." Phys. Rev. E 67, 026126

Noldus, R and Mieghem, P. 2015. "Assortativity in Complex Networks" Journal of Complex Networks. doi: 10.1093/comnet/cnv005

C. Schweik and R. English. Internet Success: A Study of Open-Source Software Commons, The MIT Press. 2012

D. Verdon and G. McGraw, "Risk analysis in software design," in IEEE Security \& Privacy, vol. 2, no. 4, pp. 79-84, July-Aug. 2004.

Walsh, M. (2011, March 11). Gov 2.0 guide to Plone. Retrieved June 23, 2016, from http://www.govfresh. com/2011/03/gov-2-0-guide-to-plone/

David A. Wagner. 2000. Static Analysis and Computer Security: New Techniques for Software Assurance. Ph.D. Dissertation. University of California, Berkeley. AAI3002306.

Plone (software). (2016, May 5). In Wikipedia, The Free Encyclopedia. Retrieved 18:20, June 23, 2016, from https://en.wikipedia.org/w/index.php?title=Plone (software)\&oldid $=718838043$

Wheeler, David A. How to Prevent the next Heartbleed. 2014-10-20. http://www.dwheeler.com/essays/ heartbleed.html

D. Wheeler and S. Khakimov. Open Source Security Census: Open Source Software Projects Needing Security Investments, Institute for Defense Analysis. 2015

Yamin, Luis Eduardo; Ghesquiere, Francis; Cardona, Omar Dario; Ordaz, Mario Gustavo. 2013. Modelacion probabilista para la gestion del 
riesgo de desastre. Washington DC ; World Bank.

http://documents.worldbank.org/curated/en/2013/07/

18100020/colombia-probabilistic-modeling-disaster-

risk-management-modelacion-probabilista-para-la-

gestion-del-riesgo-de-desastre 